\title{
Diseases from North America: focus on tick-borne infections
}

\author{
Authors: Ruvandhi R Nathavitharana ${ }^{A}$ and Jennifer A Mitty ${ }^{B}$
}

Tick-borne infections are seen throughout the United States, with varying geographical locations. Many of these infections are also increasingly seen in Europe. Certain ticks (eg Ixodes) can transmit more than one infection. Diagnosis, particularly in early infection, can be challenging and therefore knowledge of the distinguishing clinical features and epidemiology of these diseases is important. Testing for Lyme disease often causes confusion for patients and medical providers, as serological tests may be negative in early infection and conversely may be positive for years after infection. Newer tests, such as the C6 ELISA, may play a role in Lyme diagnosis. Additionally, the value of a simple blood film should not be underestimated for diagnosing babesiosis and anaplasmosis. In certain situations, empiric therapy may be required for tick-borne infections as severe illness with multiorgan failure can occur, particularly in older and immunocompromised hosts. This review describes the more commonly seen tick-borne infections: Lyme, babesiosis, anaplasmosis and Rocky Mountain Spotted Fever. More recently identified tick-borne infections, such as southern tick-associated rash illness and Borrelia miyamotoi, are also covered.

Tick-borne infections are seen throughout the United States, with varying geographical locations. Diagnosis, particularly in early infection, can be challenging and severe illness can occur. In addition, certain ticks (eg Ixodes scapularis) can transmit more than one infection. This review describes the more commonly seen tick-borne infections.

\section{Lyme disease}

In the United States, Lyme disease is the most common reportable vector-borne disease. ${ }^{1}$ It is caused by Borrelia burgdorferii, which is transmitted by the Ixodes tick. This is in contrast to Lyme disease in Europe and Asia that is primarily caused by $B$ afzelii and $B$ garinii. In the US, the Centers for Disease Control and Prevention (CDC) estimates the incidence of Lyme disease to be as high as 300,000 cases per year. ${ }^{2}$ The

Authors: Ainfectious diseases fellow, Division of Infectious Diseases, Beth Israel Deaconess Medical Center, Boston, MA, USA; Bassistant professor of medicine, Harvard Medical School, Boston, MA, USA. clinical manifestations of Lyme disease can be divided into three phases: early localised disease, which is characterised by erythema migrans (EM) with or without constitutional symptoms; early disseminated disease, which includes multiple EM lesions, cardiac and neurological involvement; and late Lyme disease, which often involves arthritis and can include neuropathy or encephalitis. ${ }^{3}$

\section{Diagnosis}

The diagnosis of Lyme disease is based upon the clinical history, epidemiological risk of exposure to Ixodes ticks, physical exam findings (especially for early localised disease) and/or serology. Patients presenting with early localised disease typically present with a characteristic rash (ie EM), which often has a central clearing or a bull's eye appearance (Fig 1). Many patients who present at this early stage of disease will have a negative serological test. Thus, early Lyme disease is usually a clinical diagnosis.

For patients presenting with later disease, a diagnosis of Lyme disease in a patient with a clinically compatible illness is supported by serological testing using a two-tier approach.

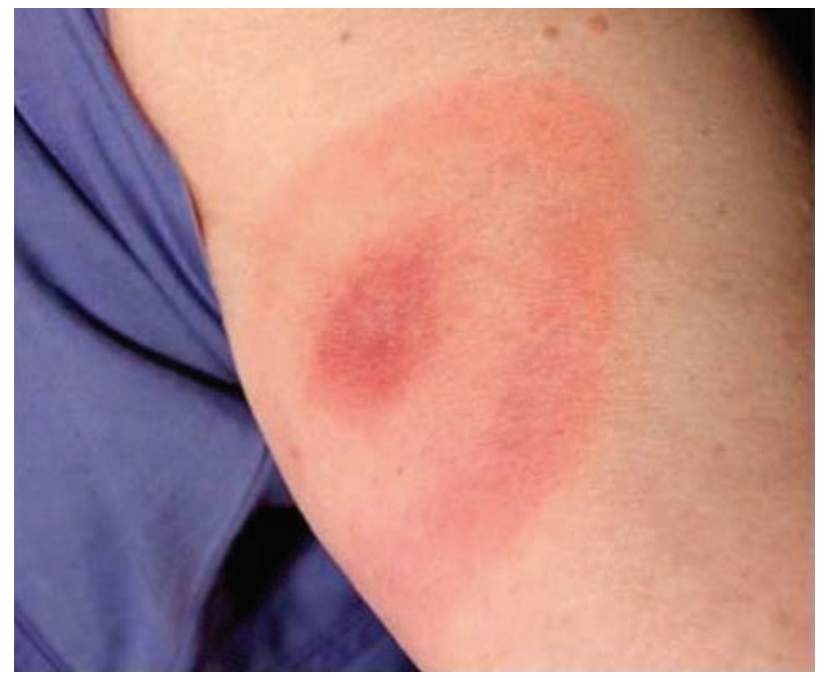

Fig 1. Typical erythema migrans rash. Image obtained from the CDC Public Health Image Library; credit: James Gathany. 
This involves a screening test, usually an enzyme-linked immunosorbent assay (ELISA) and, if positive or equivalent, a confirmatory Western blot test. For Western blot testing, CDC criteria for positivity requires $\geq 5$ bands for IgG and $\geq 2$ bands for IgM. Although the sensitivity of two-tiered serological testing is poor $(<30 \%)$ during the acute phase of infection, it is nearly $100 \%$ in patients with disseminated disease. ${ }^{4}$ Among patients who receive early antimicrobial therapy, serology may remain negative in the convalescent phase of infection due to elimination of the organism. ${ }^{1,5}$

The C6 peptide ELISA can also be used for diagnosis, although it is not routinely available. This test has a higher sensitivity and specificity than the standard ELISA but decreased specificity compared with the two-tiered test. The C6 ELISA is able to detect an early IgG response, which usually occurs before the development of $\geq 5$ bands on Western blot. ${ }^{4}$ In addition, it does not rely on the IgM response that can be associated with false-positive results. ${ }^{6}$

\section{Treatment}

Doxycycline is the mainstay of treatment, except for pregnant women and children who should receive amoxicillin or cefuroxime. Treatment duration recommendations vary. In general, 14 days of treatment is recommended for patients with EM. One study comparing 10 days of doxycycline with or without a single dose of ceftriaxone versus 20 days of doxycycline demonstrated no change in clinical outcome. ${ }^{7}$ Although 2-4 weeks is sufficient for most patients, treatment should be extended to four weeks in those presenting with arthritis. In the US, intravenous ceftriaxone is typically recommended for the treatment of patients with acute neurological Lyme disease manifested by meningitis or radiculopathy, as well as symptomatic carditis requiring hospitalisation..$^{5}$ In Europe, doxycycline is often used for the

\section{Key points}

Tick-borne infections are recognised in many parts of North America and increasingly also in Europe.

Empiric treatment should often be instituted based on clinical suspicion and epidemiological plausibility since serological tests are of limited value during early disease.

Typical laboratory abnormalities include cytopenias and liver function test abnormalities and diagnosis is often confirmed with serologies or blood films (for Babesiosis, anaplasmosis or ehrlichiosis).

Doxycycline is the mainstay of treatment for Lyme, anaplasmosis, ehrlichiosis, Rocky Mountain spotted fever (RMSF) and southern tick-associated rash illness (STARI).

Babesiosis is treated with azithromycin and atovaquone for mild disease or clindamycin and quinine for severe disease.

KEYWORDS: tick-borne, Lyme, Babesia, Anaplasma, Rocky Mountain spotted fever treatment of neurological disease since clinical trials found that doxycycline was not inferior to intravenous penicillin for neuroborreliosis. ${ }^{8}$

\section{Babesia}

Babesiosis is caused by intraerythrocytic protozoa and is most commonly due to Babesia microti in the US. ${ }^{9}$ Although Babesia species are also transmitted by the Ixodes scapularis ticks, infection has only been recognised in a limited proportion of the areas where Lyme is endemic; high incidence areas include the chain of islands off the New England coast (eg Martha's Vineyard or Nantucket). Clinical features of infection are similar to malaria and commonly include fevers, chills, sweats, myalgias and arthralgias. Laboratory findings are notable for haemolytic anaemia, thrombocytopaenia and elevated liver and kidney function tests. Patients aged over 50 years and those who are immunocompromised (eg HIV, malignancy and asplenia) are at higher risk for complicated disease. Severe babesiosis can present as acute respiratory failure, congestive heart failure, renal failure and/or disseminated intravascular coagulation. ${ }^{10}$

\section{Diagnosis}

The diagnosis is often made by microscopic identification of the organism on Giemsa stains of thin blood films. Although Babesia can be confused with malaria parasites on blood film, distinguishing characteristics can include extracellular ring forms and greater than two intraerythrocytic forms (eg the tetrad known as the Maltese cross) (Fig 2). Serology and/or polymerase chain reaction (PCR) testing can also be used for diagnosis, especially for patients with a low level of parasitaemia. $^{5}$

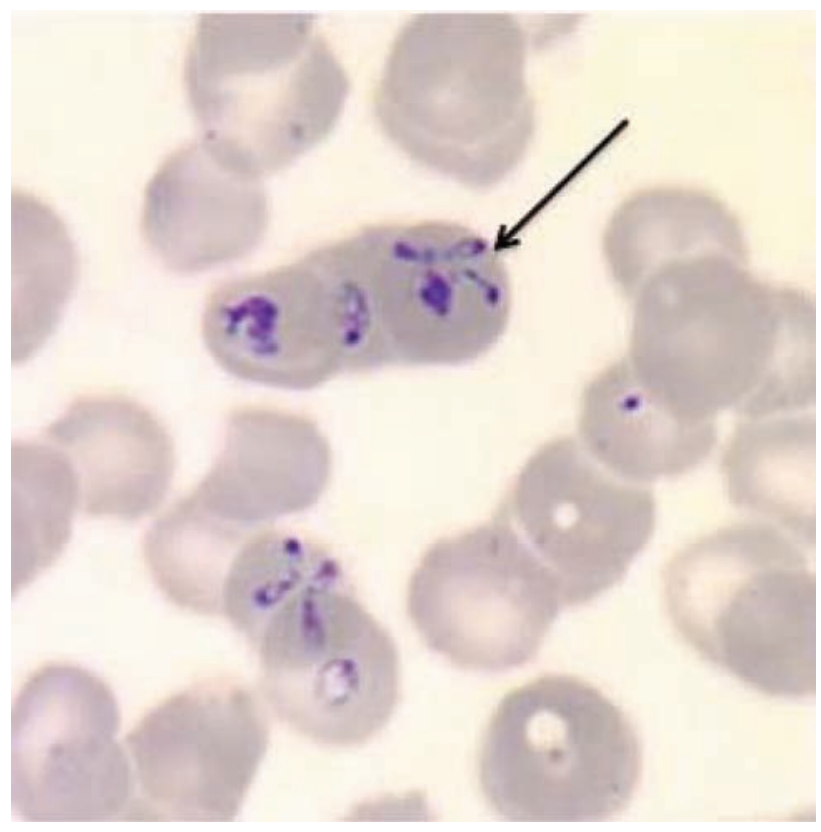

Fig 2. Babesia sp in Giemsa-stained thin blood film demonstrating the tetrad of merozoite forms known as the Maltese cross. Image obtained from CDC-DPDx. 


\section{Treatment}

A 7-10 day course of atovaquone and azithromycin is recommended for uncomplicated babesiosis. Higher doses of azithromycin (600-1,000 mg) may be used for immunocompromised hosts. For severe disease, a minimum 10-day course of clindamycin (usually given intravenously) and quinine is recommended. In addition, partial or complete red blood cell exchange transfusion may be needed for individuals with high-grade parasitaemia (>10\%), severe hemolysis, and/or renal, hepatic or respiratory compromise. These patients should be closely monitored with daily measurement of haematocrit and parasitaemia. Exchange transfusion should be continued until the level of parasitaemia has decreased to $<5 \%$.

\section{Human monocytic ehrlichiosis and human granulocytic anaplasmosis}

Ehrlichia chaffeensis causes human monocytic ehrlichiosis (HME) and Anaplasma phagocytophilum causes human granulocytic anaplasmosis (HGA). These are intracellular bacteria that grow within leukocytes. The areas of highest HGA incidence in the US are Rhode Island, Minnesota, Connecticut and Wisconsin; however, the global incidence is rising with cases being recognised in many parts of Europe. ${ }^{11}$ HME is endemic in the south-east and south-central regions of the US, such as Missouri, Oklahoma and Tennessee. ${ }^{12}$ The early symptoms of HME and HGA can mimic a viral syndrome (eg fever, chills and myalgias) and many immunocompetent patients may experience spontaneous resolution of infection. Associated laboratory abnormalities include leukopaenia, thrombocytopaenia and raised transaminases.

\section{Diagnosis}

A diagnosis is typically made through serological testing that demonstrates a four-fold change or seroconversion. Specific IgM tests are only reactive in the first 45-50 days after infection but IgG antibodies may remain positive for months or years. Additional diagnostic tests include visualisation of characteristic intracytoplasmic inclusions (morulae) on blood film (Fig 3) and/ or PCR. These latter tests are most useful for identifying early disease when antibody formation may not yet be detectable.

\section{Treatment}

Based on in vitro studies, A phagocytophilum is uniformly susceptible to tetracyclines, and doxycycline is typically the agent of choice. The duration of therapy is $7-10$ days unless Lyme co-infection is suspected. For such patients, the course should be extended to 14 days. Rifampin has been used successfully for the treatment of pregnant women and children with HGA. ${ }^{13}$

\section{RMSF}

Rocky Mountain spotted fever (RMSF) is caused by Rickettsia rickettsii which is an obligate intracellular, weakly Gramnegative coccobacillus that can be visualised on Giemsa stain. The majority of RMSF cases occur in North and South

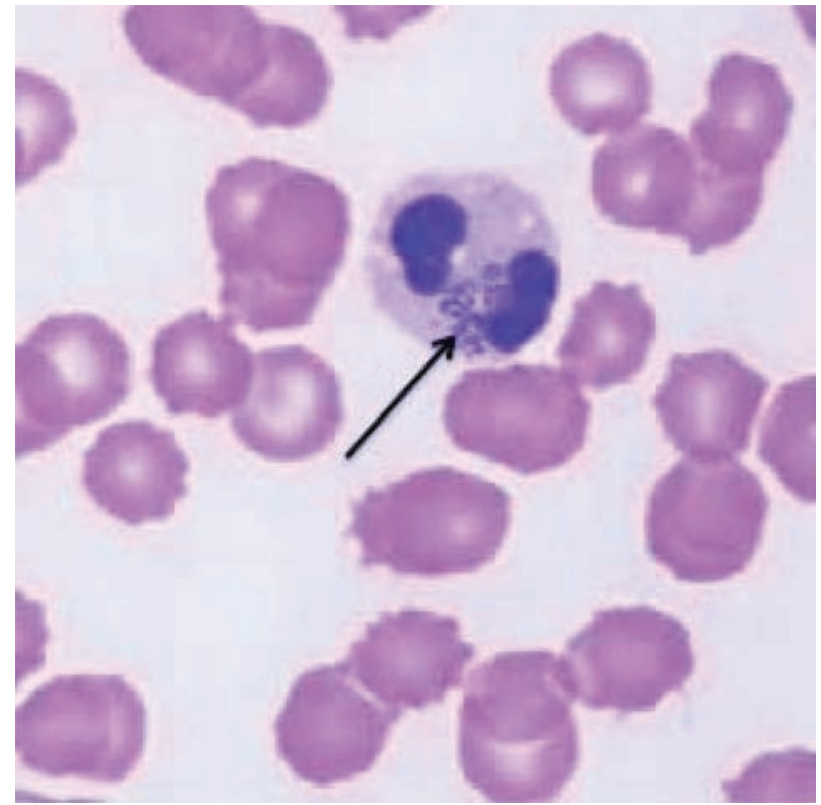

Fig 3. Ehrlichiosis evidenced by intracytoplasmic inclusions (morulae) on Giemsa-stained blood film. Image obtained from CDC-DPDx.

Carolina, Tennessee, Oklahoma and Arkansas. ${ }^{12}$ Although early symptoms mimic the viral prodrome seen with other tick-borne infections, the classic clinical triad of RMSF is fever, headache and a rash that occurs in $60-70 \%$ of patients approximately two weeks after tick exposure. The rash is due to small vessel vasculitis, and the classic finding of a rash starting at the wrists and ankles that progresses to the trunk only occurs in a minority (Fig 4).

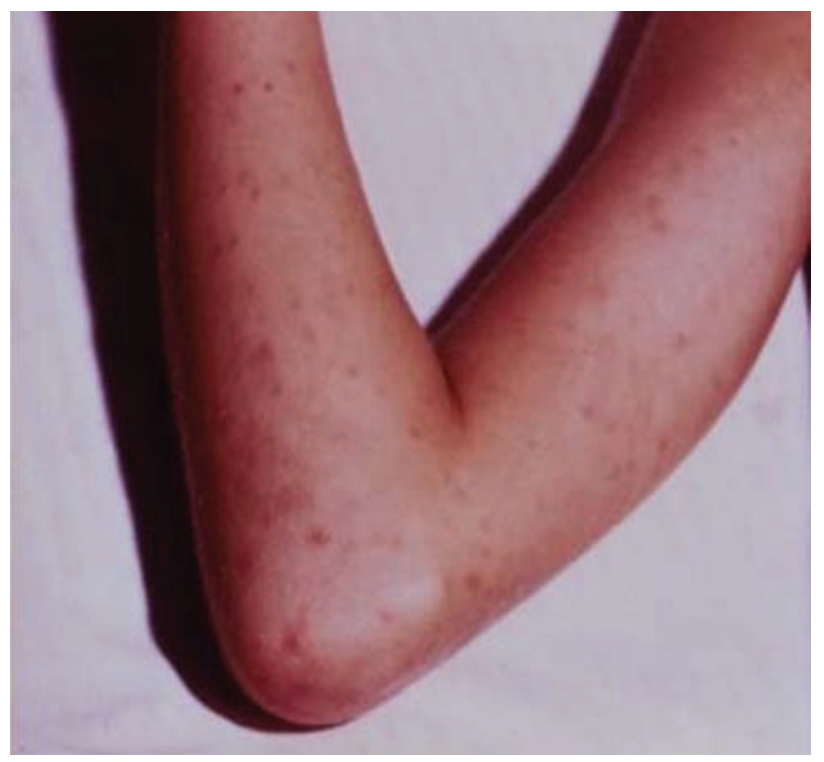

Fig 4. Typical Rocky Mountain spotted fever rash. Image obtained from the CDC Public Health Image Library. 


\section{Diagnosis}

Serology using indirect fluorescent antibody testing is the most widely available and best method. Sensitivity is poor during the first $10-12$ days but rises to $94 \%$ from days $14-21$. However, early treatment may blunt the appearance of convalescentphase antibodies. Cerebrospinal fluid demonstrates an isolated lymphocytic pleocytosis and mildly elevated protein. Direct immunofluorescence to detect $R$ rickettsii can be performed rapidly on skin biopsies with a sensitivity of approximately $70 \%$ and specificity of $100 \%$.

\section{Treatment}

Empiric treatment should be started before laboratory confirmation is obtained due to increased risk of mortality with treatment delays. Thus, initial treatment decisions should be guided by clinical suspicion and epidemiological plausibility. Doxycycline is the treatment of choice and treatment duration is typically 5-7 days. The only other recommended agent for those with severe hypersensitivity or pregnant women in the first or second trimesters is chloramphenicol. ${ }^{14}$

\section{STARI}

The presentation of EM-like skin lesions in patients from areas not associated with Ixodes ticks should raise concern for southern tick-associated rash illness, also known as STARI. This is associated with bites from the Lone Star tick, Amblyomma americanum. Treatment consists of a 10-day course of doxycycline. ${ }^{15}$

\section{B miyamotoi}

A recently identified spirochete called B miyamotoi has been associated with infections both in North America and Europe. ${ }^{16}$ Clinical manifestations include a viral-like illness, although meningoencephalitis has been reported. ${ }^{17,18}$ Although diagnostic testing for this organism is not routinely available, serology and PCR testing can be performed in certain labs. Doxycycline and/ or ceftriaxone are effective treatments.

\section{Prevention}

The best ways to avoid these tick-borne infections are using DEET-containing insect repellant, wearing long-sleeved shirts or trousers, and/or clothes treated with permethrin. In addition, showering and performing tick checks should be done after exposure. A single $200 \mathrm{mg}$ dose of doxycycline was shown to be $87 \%$ effective in preventing Lyme disease if administered within 72 hours in a randomised control trial. ${ }^{19}$ This strategy is recommended for individuals who have a tick that has been attached for 36 hours and/or those who remove an engorged deer tick. ${ }^{5}$ Although this preventative approach is often used, a meta-analysis showed that 50 people bitten by a deer tick would need prophylaxis to prevent one case of EM. ${ }^{20}$

\section{References}

1 Shapiro ED. Clinical practice. Lyme disease. $N$ Engl J Med 2014;370:1724-31.

2 Kuehn BM. CDC estimates 300,000 US cases of Lyme disease annually. JAMA 2013;310:1110.

3 Steere AC. Lyme disease. N Engl J Med 1989;321:586-96.

4 Steere AC, McHugh G, Damle N, Sikand VK. Prospective study of serologic tests for Lyme disease. Clin Infect Dis 2008;47:188-95.

5 Wormser GP, Dattwyler RJ, Shapiro ED et al. The clinical assessment, treatment, and prevention of lyme disease, human granulocytic anaplasmosis, and babesiosis: clinical practice guidelines by the Infectious Diseases Society of America. Clin Infect Dis 2006;43:1089-134.

6 Halperin JJ, Baker P, Wormser GP. Common misconceptions about Lyme disease. Am J Med 2013;126:264.e1-7.

7 Wormser GP, Ramanathan R, Nowakowski J et al. Duration of antibiotic therapy for early Lyme disease. A randomized, double-blind, placebo-controlled trial. Ann Intern Med 2003;138:697-704.

8 Karlsson M, Hammers-Berggren S, Lindquist L, Stiernstedt G, Svenungsson B. Comparison of intravenous penicillin $\mathrm{G}$ and oral doxycycline for treatment of Lyme neuroborreliosis. Neurology 1994;44:1203-7.

9 Vannier E, Gewurz BE, Krause PJ. Human babesiosis. Infect Dis Clin North Am 2008;22:469-88,viii-ix.

10 Hatcher JC, Greenberg PD, Antique J, Jimenez-Lucho VE. Severe babesiosis in Long Island: review of 34 cases and their complications. Clin Infect Dis 2001;32:1117-25.

11 Dumler JS, Choi KS, Garcia-Garcia JC et al. Human granulocytic anaplasmosis and Anaplasma phagocytophilum. Emerg Infect Dis 2005;11:1828-34.

12 Chapman AS, Bakken JS, Folk SM et al. Diagnosis and management of tickborne rickettsial diseases: Rocky Mountain spotted fever, ehrlichioses, and anaplasmosis - United States: a practical guide for physicians and other health-care and public health professionals. MMWR Recomm Rep 2006;55:1-27.

13 Bakken JS, Dumler S. Human granulocytic anaplasmosis. Infect Dis Clin North Am 2008;22:433-48,viii.

14 Chen LF, Sexton DJ. What's new in Rocky Mountain spotted fever? Infect Dis Clin North Am 2008;22:415-32,vii-viii.

15 Armstrong PM, Rich SM, Smith RD, Hartl DL, Spielman A, Telford SR 3rd. A new Borrelia infecting Lone Star ticks. Lancet 1996;347:67-8.

16 Krause PJ, Narasimhan S, Wormser GP et al. Borrelia miyamotoi sensu lato seroreactivity and seroprevalence in the northeastern United States. Emerg Infect Dis 2014;20:1183-90.

17 Krause PJ, Narasimhan S, Wormser GP et al. Human Borrelia miyamotoi infection in the United States. N Engl J Med 2013;368:291-3.

18 Gugliotta JL, Goethert HK, Berardi VP, Telford SR 3rd. Meningoencephalitis from Borrelia miyamotoi in an immunocompromised patient. N Engl J Med 2013;368:240-5.

19 Nadelman RB, Nowakowski J, Fish D et al. Prophylaxis with singledose doxycycline for the prevention of Lyme disease after an Ixodes scapularis tick bite. N Engl J Med 2001;345:79-84.

20 Warshafsky S, Lee DH, Francois LK, Nowakowski J, Nadelman RB, Wormser GP. Efficacy of antibiotic prophylaxis for the prevention of Lyme disease: an updated systematic review and meta-analysis. J Antimicrob Chemother 2010;65:1137-44.

Address for correspondence: Dr RR Nathavitharana, Division of Infectious Diseases, Beth Israel Deaconess Medical Center, 110 Francis Street, Suite GB, Boston, MA 02215, USA.

Email: rnathavi@bidmc.harvard.edu 